\title{
SUBSURFACE IMAGING USING GROUND PENETRATING RADAR FOR UNDERGROUND OIL AND MINERALS EXPLORATION
}

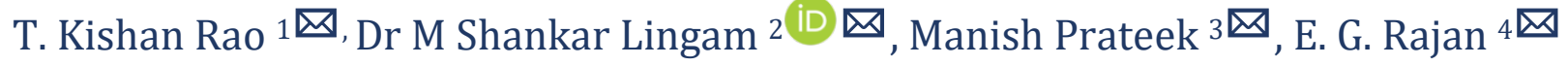 \\ ${ }^{1}$ Research Scholar MG-NIRSA University of Mysore, India \\ 2 Research Associate NIRDPR, Hyderabad, India \\ 3 Professor and Dean University of Petroleum \& Energy Studies Dehradun, Uttarakhand, India \\ ${ }^{4}$ University of Petroleum \& Energy Studies Dehradun, Uttarakhand, India, Hon. Director MG- \\ NIRSA, India
}

DOI: https://doi.org/10.29121/IJOEST.v4.i6.2020.139

Article Type: Research Article

Article Citation: T. Kishan Rao, Dr M Shankar Lingam, Manish Prateek, and E. G. Rajan. (2020).

SUBSURFACE IMAGING USING GROUND PENETRATING RADAR FOR UNDERGROUND OIL AND MINERALS EXPLORATION. International Journal of Engineering Science Technologies, 4(6), 71-79. https://doi.org/10.29121/IJOEST.v 4.i6.2020.139

Received Date: 20 November 2020

Accepted Date: 31 November 2020

Keywords:

Oil Exploration

Ground Penetrating Radar

Vertical Seismic Profiling

\begin{abstract}
An oil well drilling company contractor drills an oil well will as per specific requirement like the depth. It has been estimated that cost to drill an oil well is about \$17.01/ Barrels-of-Oil-Equivalent (BOE). More specifically, cost for drilling an onshore well is $\$ 13.38 / \mathrm{BOE}$ and for drilling an offshore well is $\$ 49.54 / \mathrm{BOE}$. Based on some statistics one out of five rigs drilled in an area yields oil recovery. This means $\$ 20$ million has to be spent for prospecting and locating one oil well. The question that arises now is whether it is worth trying all possibilities of reducing the cost of locating an oil well to $\$ 4$ million and save $\$ 16$ million. Well, the research presented in this paper is aimed at showing such a possibility.
\end{abstract}

\section{INTRODUCTION}

Three-dimensional digital image processing has numerous applications in the fields of medical imaging, seismology, geomorphology, remote sensing and material sciences. Based on the requirement to solve a problem of interest, one would classify features of three-dimensional images and apply relevant algorithms. For example, the quantitative analysis of geomorphological structures in GPR radargrams and other similar images requires extraction of volumes and shapes of geological constructs like oil reserves, ores and minerals, and encapsulated gems, gold and diamonds present below the surface of earth. In order to do this, there is a need for an aerial survey of the area of interest for locating the land for scanning using ground penetrating radar. 


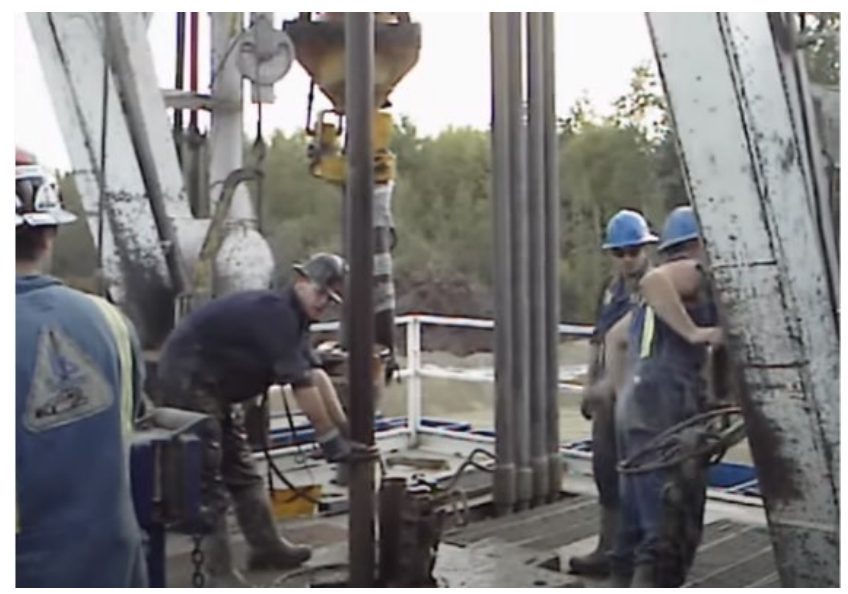

For example, drilling an oil well is done by a contractor. The contractor would operate as per the contract terms, like depth of the well and width of the bore. The contractor charges the beneficiary on per day or else per foot rate of drilling. In 2007 it was costing $\$ 4$ million to drill a well and \$3.9 million to drill a gas well. The average cost to drill a well has been $\$ 574 /$ foot of depth. The average cost to drill oil or gas well property in USA was $\$ 17.01 /$ The onshore development cost was $\$ 13.38 / \mathrm{BOE}$ and offshore development cost was $\$ 49.54 / \mathrm{BOE}$. Based on some statistics one out of five rigs drilled in an area yields oil recovery. This means $\$ 20$ million has to be spent for prospecting and locating one oil well. The question that arises now is whether it is worth trying all possibilities of reducing the cost of locating an oil well to $\$ 4$ million and save $\$ 16$ million. The research presented in this paper is aimed at showing such a possibility.

\subsection{OBJECTIVE OF THE RESEARCH}

Major objectives of this intended research are:

1) To develop new methodologies for data acquisition, processing, interpretation

2) To integrate multiple technologies focusing VSP (Vertical Seismic Profiling) methods and appropriate interpretation in order to locate new oil reserves.

3) To make use of VSP (Vertical Seismic Profiling) methodology for prospecting minefield development and management for maximum economic recovery of various minerals and gems.

\subsection{SUB-OBJECTIVES OF THIS RESEARCH}

1) To device methodologies for collecting data from underground of the earth crust. Data acquisition sensors like Synthetic Aperture Radar (SAR) or Ground Penetrating Radar (GPR) and Microwave pulse transmitters could be used to achieve this. Other seismographic techniques could also be used alternatively or in association with SAR/ GPR.

2) Multiple Sensor Fusion is the next part. Data collected from various sensors could be integrated as a data set from which quite a lot of information about the subsurface elements could be interpreted.

3) Fast and reliable algorithms for extracting information from the data set is the major R\&D component involved in this research work.

4) To develop a state-of-the-art technology to use the developed tools for detecting oil, minerals and gems reserve in a particular area from which genuine and reliable data set has been collected.

5) However, the material presented in this paper is restricted to the fundamental research carried out as to how to prospect a field of interest and how to obtain a 3D radargram for the purpose of data analysis.

\section{METHODOLOGY TO BE USED IN PROSPECTING A FIELD OF INTEREST}

While radar systems work in a free-space environment, GPRs work in media that exhibit electromagnetic properties, which are frequency-dependent. So, the reflected electromagnetic energy shows dispersive effects. Radar 
systems are used to detect targets at ranges of hundreds of kilometers, but in the case of GPR, targets at ranges of some meters only are detected because of limitation in the transmitted power and attenuation of the reflected signal. Moreover, resolution of GPR is very much limited to centimetres or metres (based on the active frequencies, which are smaller when compared to those used in tactical radar systems). In any case, one has to employ frequencies ranging from tens of $\mathrm{MHz}$ to some $\mathrm{GHz}$ in order to achieve good spatial resolution of the reflected data. Different GPR configurations are used in land surveys and their choice is made based on different applications like (i) the type of exploration to be made, (ii) the type of targets to be detected, and (iii) the extent of region to be explored. For example, the region to be explored is studied initially by an aerial survey. Fig. 1 shows the image of an area obtained using a drone camera. After visualizing the real field image obtained using a drone camera, a geomorphologist would be able to decide on the fact whether the concerned area is worth to be prospected.



Figure 1: Aerial visualization of an area to be prospected using a drone camera Let us consider an oil field model shown in Fig. 2 as a rectangular / hexagonal pattern.

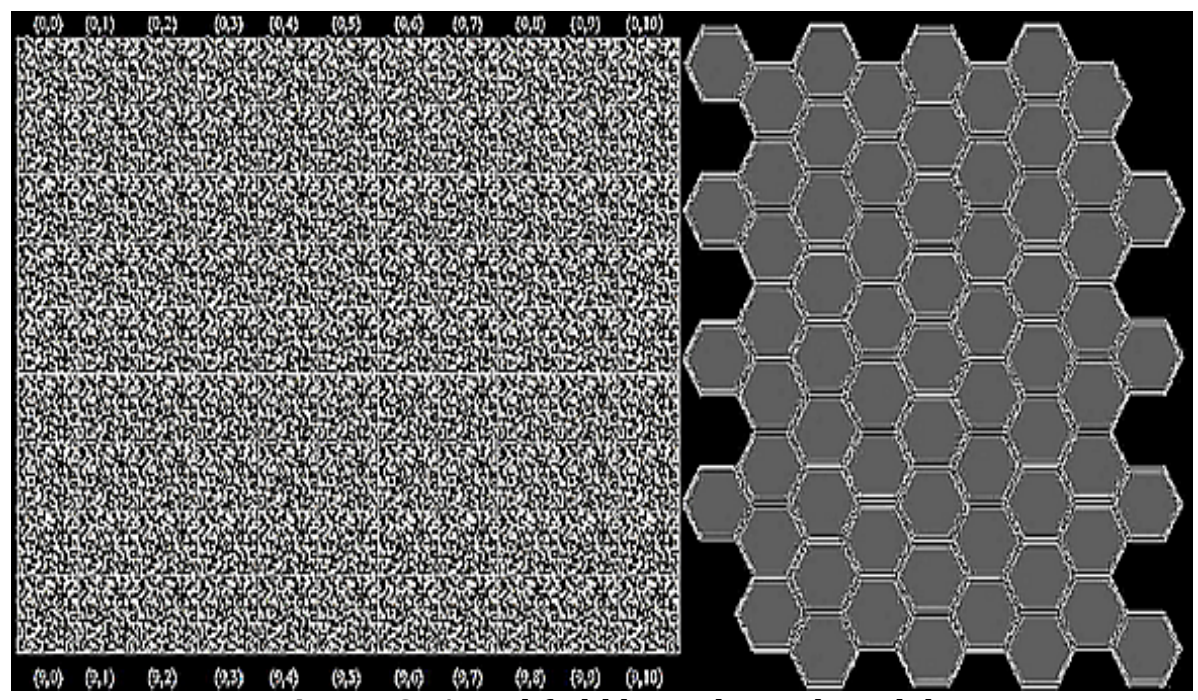

Figure 2: An oil field hypothetical model

Rectangular model is generally preferred to avoid operational difficulties. The rectangular field model is divided into a matrix of $11 X 9$ sub regions. Scanning of a minefield is carried out using a GPS controlled autonomous mobile robot (AMR) system consisting of a ground penetrating radar and image acquisition system. Serpentine scanning is used for prospecting mine fields. Fig. 3 shows the scanning types generally used by an AMR. 

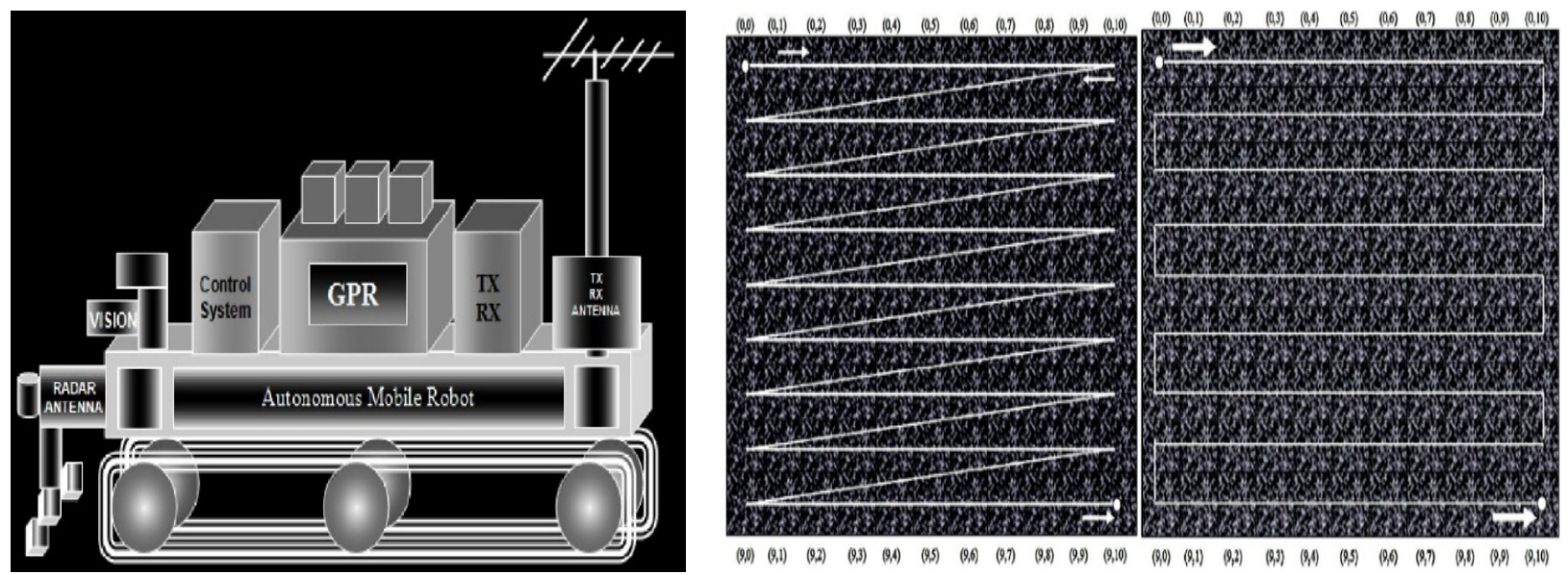

Figure 3: Scanning types used by an autonomous mobile robot system

Ground Penetration Radar (GPR) is used for excitation. Excitation is applied at appropriate locations, preferably at the centers of the sub divided regions. Fig. 4 shows excitation process as a serpentine scanning of a rectangular subsampled field model.
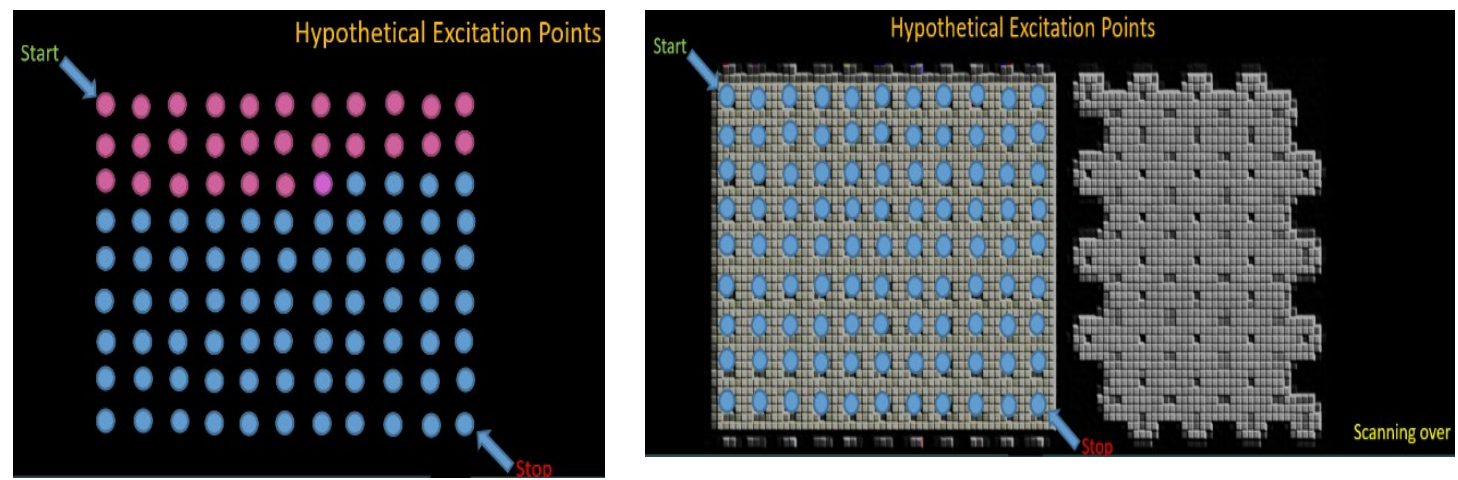

Figure 4: Excitation process as a serpentine scanning of rectangular field model

Area of a sub divided region determines the resolution. The intensity of excitation depends on many factors but mainly on the required depth of penetration. A short duration pulse is applied as excitation and the echo received and registered for further analysis. With reference to the mine field model shown, one would obtain 99 echoes in the form of one-dimensional characteristic signals. The hypothetical excitation points are shown in Fig. 5.

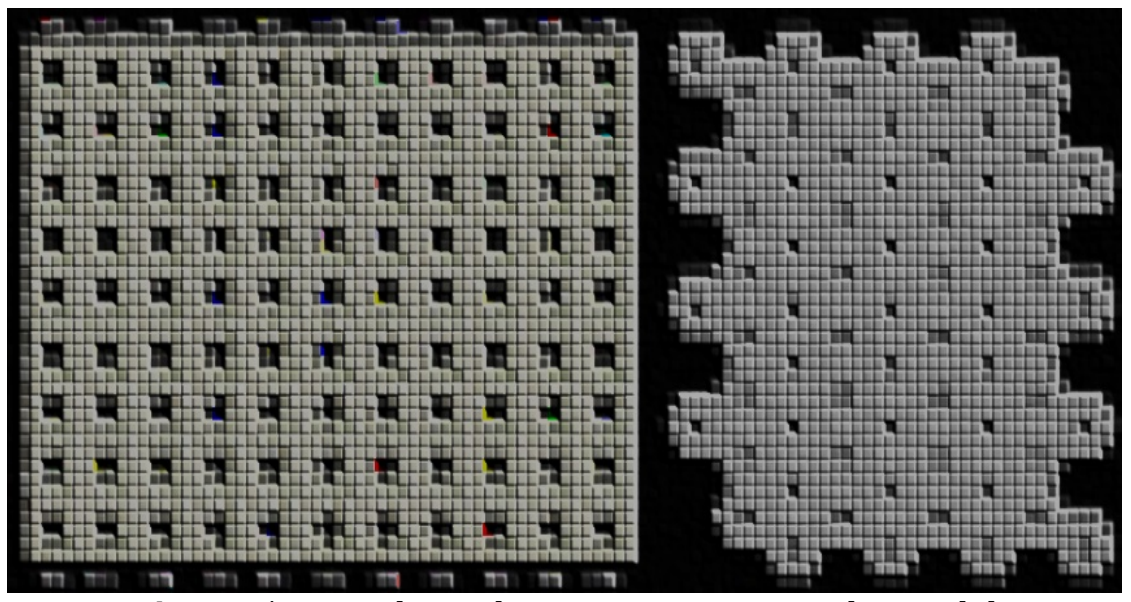

Figure 5: Hypothetical excitation points in the model

The received signal from an excitation point both in the analog and discrete form is shown in Fig. 6. 

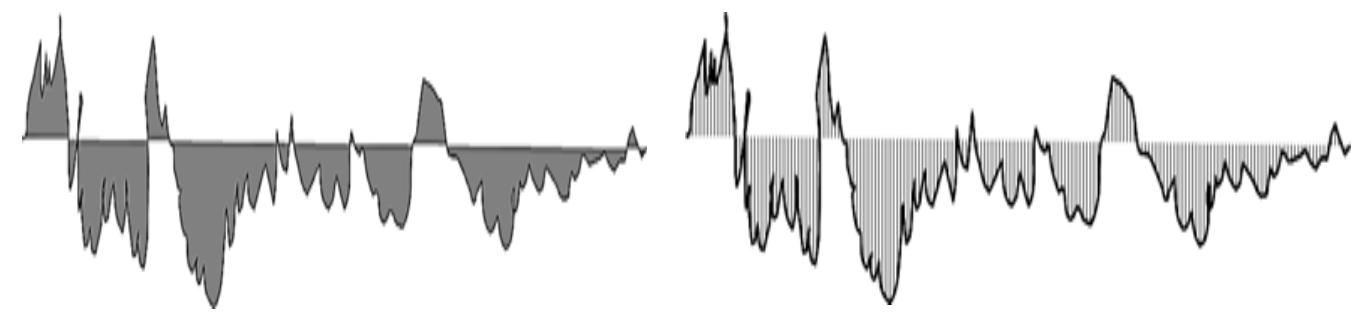

Figure6: Acquired radar echo from under the earth surface in analog and discrete form

The sampling of the analog signal is carried out with the Nyquist frequency without causing any loss in the vital information related to characteristic echo due to various sub surface elements. Fig. 7 shows ensemble of 1-D signals (echoes spatially distributed) assumed for the hypothetical field model, its solid model and sectional views.

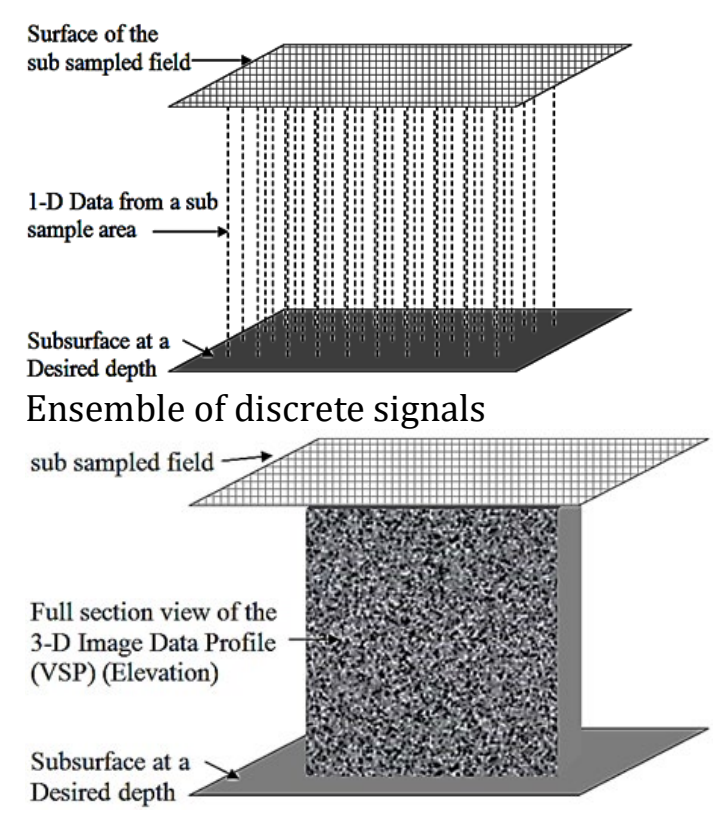

Full section of the elevation view

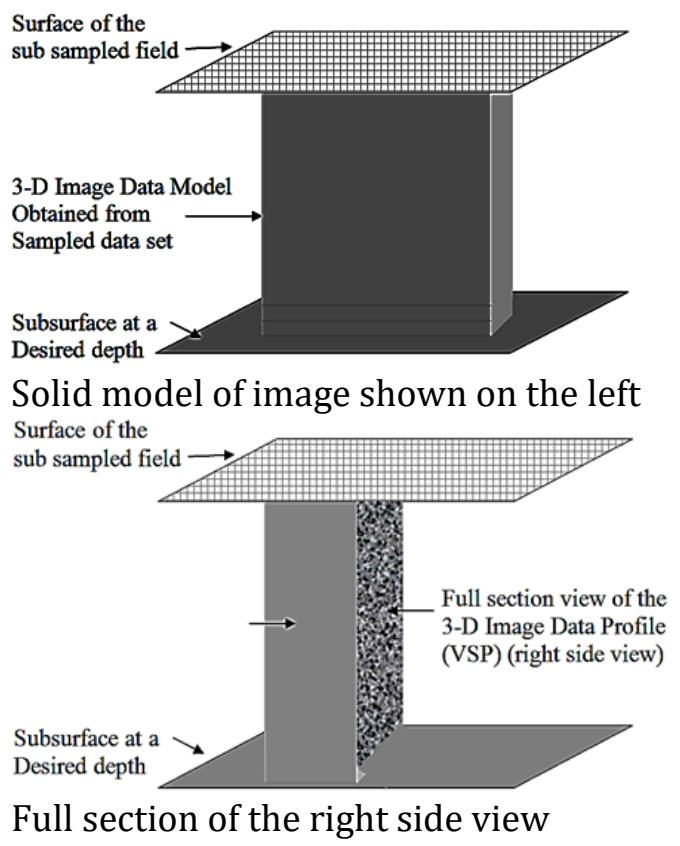

Full section of the right side view

Figure 7: Ensemble of discrete signals, solid model and its sectional views

The ensemble of discrete signals is viewed as a sparsed 3D data. One can dilate this sparsed data in order to get a solid model. Fig. 7 shows the solid model of the sparsed data, elevation and right side full section views of the VSP Stack. VSP refers to Vertical Seismic Profile. VSP is also known as sectional profile. HSP refers to Horizontal Seismic Profile. HSP is also known as depth slice or time slice. VSP stack is generally used to predict oil and other mineral reserves, whereas HSP is used for archaeological survey. Fig. 8 shows a hypothetical VSP and HSP.

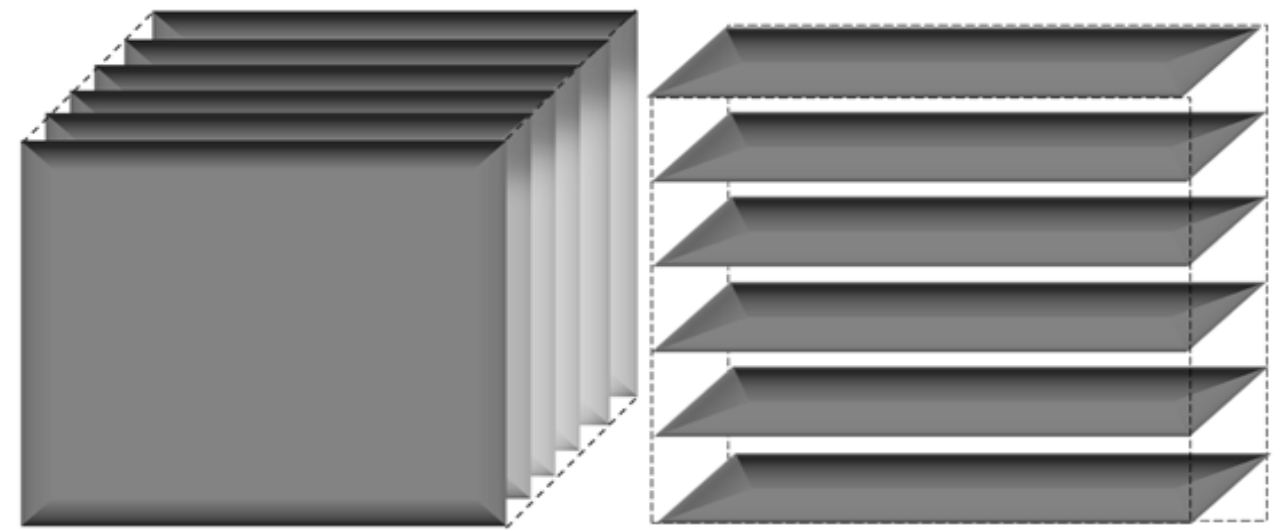

Figure 8: A sample VSP and HSP 
Once VSP or HSP is obtained, one can use 3D image processing procedures to obtain hidden evidence for locating subsurface components of interest. Discrete lines of GPR data denote a sectional subsurface view. Multiple lines of GPR data collected over a specific area are used to obtain relevant three-dimensional images. Data could be viewed either as three-dimensional blocks, or as horizontal/vertical slices. Horizontal slices (known as "depth slices" or "time slices") denote plan view plots separating specific depths. Time-slicing is a standard practice adopted by archaeologists, because horizontal patterning mostly indicates many evidences related to cultural activities.

A survey was made in November 1993 using analog GPR equipment on Nutubaru Kofun burial site in Shintomi Machi, Ishikawa, Japan and 3D depth slices obtained. Fig. 9 shows a large round anomaly found at a depth as reflections from the moat of a destroyed burial mound dating from the 6-7th century AD. The full description of the history of this mound could be seen in various literature. The authors acknowledge Geophysical Archaeometry Laboratory Inc., 20014 Gypsy Ln Woodland Hills, California 91364 for providing details about the visual material related to GPR applications presented in this paper. For details visit their web site: http://www.gpr-survey.com/.

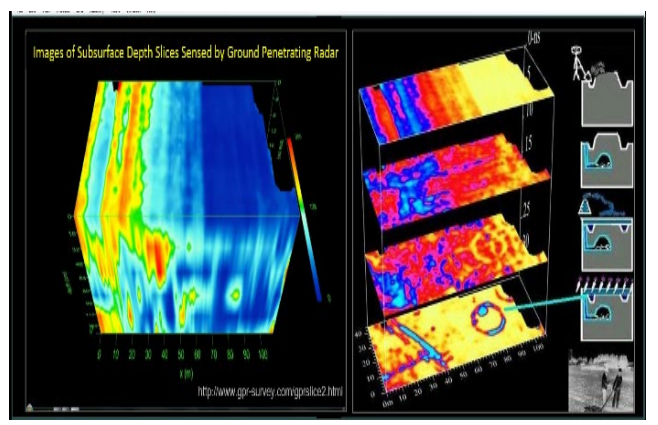

Subsurface depth slices obtained using a GPR

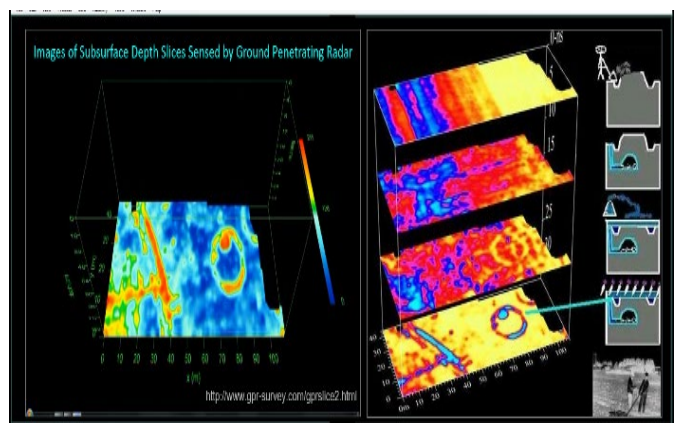

Radar echo from the mote of a burial mound

Figure 9: HSP slices obtained using a GPR at a burial site in Japan

Figure 10 shows actual HSP slices and the corresponding image of the excavated burial site in Japan.

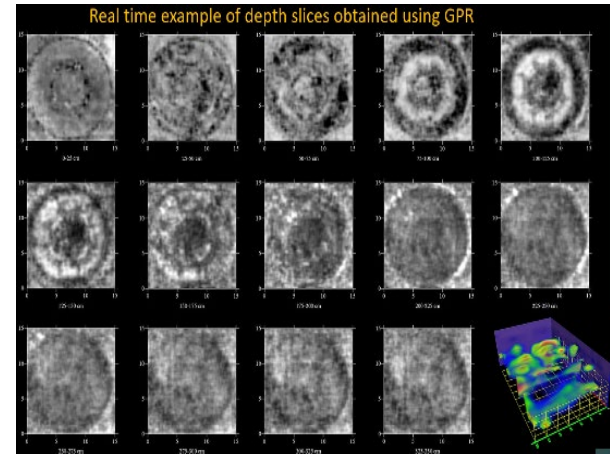

Fourteen depth slices

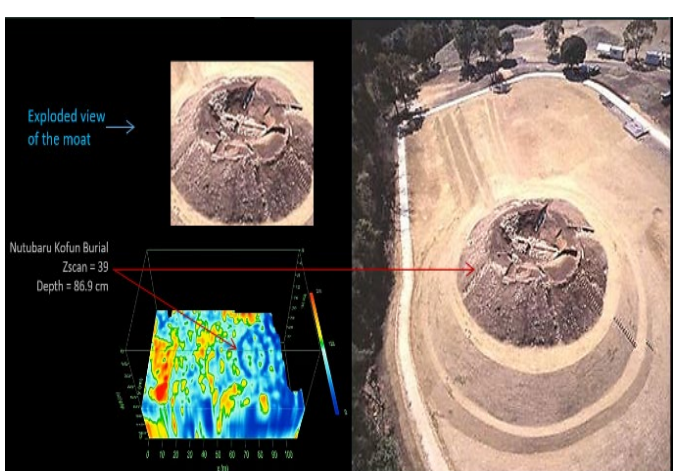

Excavated site image

Figure 10: Fourteen depth slices of the HSP obtained using a GPR at a burial site in Japan

\section{GROUND PENETRATING RADAR AND RADARGRAM ACQUISITION}

An archaeological site is surveyed by a mobile GPR at a finite number of locations at the site denoted by $\mathrm{x}$ and $\mathrm{y}$, and radar excitation pulses fired through the ground surface. The reflected echoes due to individual pulses representing the depth of $3^{\text {rd }}$ dimension, say $\mathrm{z}$ are collected and recorded from which the 3D radargram image is developed. In order to make such 3D images, various signal processing techniques are used. Signal processing operations are certain mathematical models and they carry out noise filtering and signal integration on raw radar pulse data. Some of the basic GPR data processing operations are briefly explained below.

\section{Post Processing Gain Improvement}

Usually, GPR data are 16-bit discrete sequences. The discrete data samples obtained from GPR echoes vary from -32768 to +32767 . Amplitudes of the reflected echoes from just below ground surface to an intended depth would 
vary from a maximum to a minimum. The resistance offered to echoes that travel from a depth to the surface would be maximum so that the echoes from various layers of earth would be weaker and weaker depending on the depth. This calls for gain improvement, possibly an automatic gain control has to be used in order to study the characteristic reflections from various scatterers from under the ground.

\section{Drift Removal}

The gain corrected raw radargrams would often be corrupted by different kinds of noise and this would shift the DC base line above ' 0 '. In such a case, one would use appropriate filters, like wiener filter, anisotropic diffusion filter, log-normal filter, depending on the type of noise like additive or multiplicative noise or speckle noise, present in the radargram. Since the base line shifted radargram will not appear as true visual model of the actual subsurface components, DC restoration is done on the radargram with the help of denoising filters.

\section{Background Removal}

The echoes obtained from various depths not only due to scatters of specific interest but also due to soil, rock and other scatterers of no interest. Such echoes are called background noise. The echoes from scatterers are interest are called signatures. Background removed signatures would yield a meaningful visual presentation due to scatterers of interest.

\section{Migration}

Moving a GPR along a profile line would cause the profile of echoes from a particular scatterer to look like an inverted hyperbola. This is due to the fact that the GPR antenna would not only receive echoes from a scatterer when the antenna is just above it but also receive echoes from the same scatterer even after the GPR moving away from it. In addition, noise would also corrupt the presentation of the inverted hyperbola. In such a case, an operation called migration is carried out as a signal conditioning measure. The process of migration is caried out by fitting a hyperbola corresponding to a scatterer by adding up all energy values along the hyperbola and keeping this addition right at the top of the inverted hyperbola. This is done for every point in the radargram. The net result is to break the hyperbolas to smaller point scatterers. The next step after migration, the radargram should be processed to obtain a meaningful image from this data. Time slice presentation is the most useful image for archaeological applications. Time slices or HSPs represent maps of amplitudes of recorded reflections across a site at a specified time or depth. One could follow the following technique to create the most meaningful images for archaeological applications.

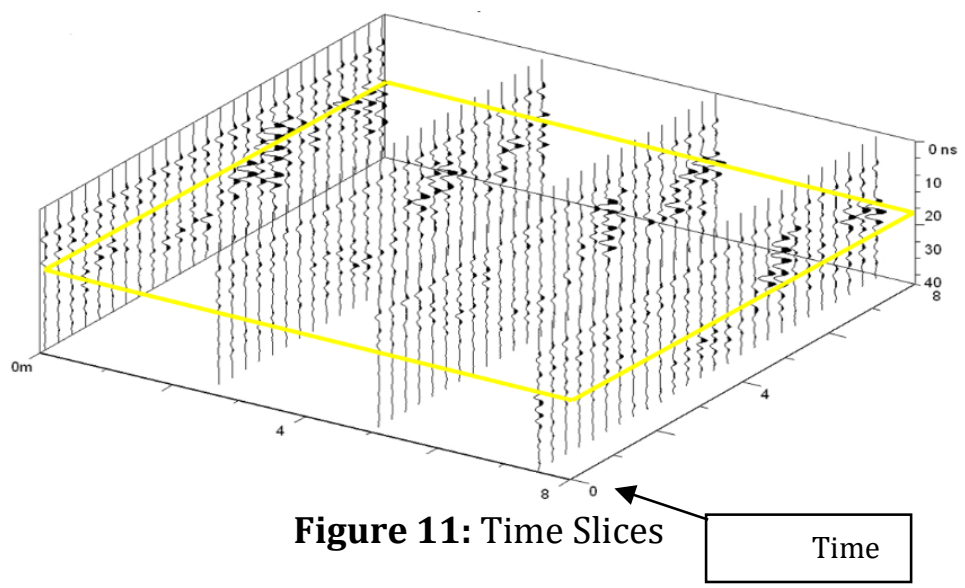

\section{Processing of 3-D images using cellular logic methods}

There is another novel paradigm called 'Cellular Logic Array Processing (CLAP)' introduced by E. G. Rajan, in which one can develop fast algorithms for processing digital images. Cellular Logic Array Processing is a logicomathematical framework developed using the fundamental notions of Markov's 'Normal Algorithms' and von Neumann's 'Cellular Automata'. Normal algorithm is an idealized serial processor like a Turing machine and a cellular automaton is an idealized parallel processor. CLAP is a logical technique of realizing cellular automata using normal algorithms.

\section{Expected Research Impact}

The research output would open up new avenues for developing a prototype product and whose future versions would be used in various 3-D image processing applications like 'Medical Image Processing', 'Mineral Exploration', 
Subsurface Imaging Using Ground Penetrating Radar for Underground Oil and Minerals Exploration

'Archeological Excavations and 'Defense Applications'. Some of the imminent benefits of this research are envisaged as outlined below.

\section{Societal benefits}

- Radiologists, medical professionals can identify various features from 3-D images for diagnostic purposes.

- Prediction of damaged tissues/organs in less time without going for surgery or biochemical test.

- Useful for researchers in analyzing the 3-D structures in various fields like oncology, geology, zoology, aviation, geophysics, and protein modeling etc.

- It is useful for detecting un-exploded landmines, where layer wise 2-D images of particular land are obtained by Ground Penetrating Radar devices.

- It is useful for knowing the position of archeological objects under the ground so that objects are not damaged while excavating from the ground.

\section{Economic benefits}

- Oil companies spend lots of money in digging wells to explore the presence of oil. The 2-D image of the layers of the earth can be obtained by using ground penetrating radar (GPR) device. The sequence of these 2-D images can be reconstructed to form a 3-D image called Vertical Seismic Profile (VSP) and such 3-D images can be processed to obtain information about the presence of oil or other elements of interest. By using this technique oil companies can have a priori knowledge about a site and then go for a rig which means saving of money.

- Similarly, medical professionals and other people like 3-D film producers who make use of 3-D image processing and analysis can get their jobs done effectively, reliably and with reduced cost.

\section{SOURCES OF FUNDING}

This research received no specific grant from any funding agency in the public, commercial, or not-for-profit sectors.

\section{CONFLICT OF INTEREST}

The author have declared that no competing interests exist.

\section{ACKNOWLEDGMENT}

None.

\section{REFERENCES}

[1] Jean Serra; Cube, cube-octahedron or rhomb-dodecahedron as bases for 3-D shape descriptions, Advances in visual form analysis, World Scientific 1997, 502-519.

[2] Wuthrich, C.A. and Stucki, P.; An algorithm comparison between square- and hexagonal based grids; Graphical Models and Image Processing, 53(4), 324-339,1991.

[3] Reinhard Klette and Azriel Rosenfeld; Digital Geometry: Geometric methods for picture analysis; Elsevier, 2004.

[4] B. Nagy; Geometry of Neighborhood sequences in hexagonal grid; Discrete Geometry of computer imagery; LNCS-4245, Springer.

[5] Tristan Roussillon, Laure Tougne, and Isabelle Sivignon; What Does Digital Straightness talk about Digital Convexity?

[6] Edward Angel, "Interactive Computer Graphics- a top-down approach with OpenGL", Second Edition, Addison Wesley, 2000.

[7] S.W. Zucker, R.A. Hummel, "A Three-Dimensional Edge Operator", IEEE Trans. On PAMI, Vol. 3, May 1981.

[8] Jürgen, H., Manner, R., Knittel, G., and Strasser, W. (1995). Three Architectures for Volume Rendering. International Journal of the Eurographics Association, 14, 111-122

[9] Dachille, F. (1997). Volume Visualization Algorithms and Architectures. Research Proficiency Examination, SUNY at Stony Brook

International Journal of Engineering Science Technologies 
[10] Günther, T., Poliwoda, C., Reinhart, C., Hesser, J., and Manner, J. (1994). VIRIM: A Massively Parallel Processor for Real-Time Volume Visualization in Medicine. Proceedings of the 9th Eurographics Hardware Workshop, 103-108

[11] Boer, M.De, Gröpl, A., Hesser, J., and Manner, R. (1996). Latency-and Hazard-free Volume Memory Architecture for Direct Volume Rendering. Eurographics Workshop on Graphics Hardware, 109-119

[12] Swan, J.E. (1997). Object Order Rendering of Discrete Objects. PhD. Thesis, Department of Computer and Information Science, The Ohio State University

[13] Yagel, R. (1996). Classification and Survey of Algorithms for Volume Viewing. SIGGRAPH tutorial notes (course 34)

[14] Law, A. (1996). Exploiting Coherency in Parallel Algorithms for Volume Rendering. PhD. Thesis, Department of Computer and Information Science, The Ohio State University

[15] Ray, H., Pfister, H., Silver, D., and Cook, T.A. (1999). Ray Casting Architectures for Volume Visualization. IEEE Transactions on Visualization and Computer Graphics, 5(3), 210-233

[16] Yagel, R. (1996). Towards Real Time Volume Rendering. Proceedings of GRAPHICON' 96, 230-241

[17] Kaufman, A.E. (1994). Voxels as a Computational Representation of Geometry. in The Computational Representation of Geometry SIGGRAPH '94 Course Notes

[18] Lacroute, P., and Levoy, M. (1994). Fast Volume Rendering Using a Shear-Warp Factorization of the Viewing Transform. Computer Graphics Proceedings Annual Conference Series ACM SIGGRAPH, 451-458.

[19] Sutherland, I.E., Sproull, R.F., and Schumaker, R.A. (1974) A Characterization of Ten Hidden Surface Algorithms. ACM Computing Surveys, 6(1), 1-55

[20] Roberts, J.C. (1993). An Overview of Rendering from Volume Data including Surface and Volume Rendering. Technical Report 13-93*, University of Kent, Computing Laboratory, Canterbury, UK.

[21] Frieder, G., Gordon, D., and Reynolds, R.A. (1985). Back-to-Front Display of Voxel-Based Objects. IEEE Computer Graphics and Applications, 5(1), 52-60

[22] Westover, A.L. (1991). Splatting: A Parallel Feed-Forward Volume Rendering Algorithm. Ph.D. Dissertation, Department of Computer Science, The University of North Carolina at Chapel Hill

[23] Zwicker, M., Pfister., H., Baar, J.B. and Gross M. (2001). Surface Splatting. In Computer Graphics SIGGRAPH 2001 Proceedings, 371-378

[24] Nulkar, M., and Mueller, K. (2001). Splatting with Shadows. International Workshop on Volume Graphics 2001,35-50

[25] J. Krüger and R. Westermann, Acceleration Techniques for GPU-based Volume Rendering, Proceedings of the 14th IEEE Visualization 2003 (VIS'03), 38-43.

[26] Markus Hadwiger, Joe M. Kniss, Christ of Rezk-salama, Daniel Weiskopf, and Klaus Engel. Real time Volume Graphics. A. K. Peters, Ltd., USA, 2006.

[27] Goodman, D., Nishimura, Y., Hongo, H and Noriaki N., 2006. Correcting for topography and the tilt of the GPR antenna, Archaeological Prospection, 13: 157-161.

[28] Goodman, D., Y. Nishimura, and J. D. Rogers, 1995. GPR time slices in archaeological prospection: Archaeological Prospection, 2:85-89.

[29] Goodman, D., 1994. Ground-penetrating radar simulation in engineering and archaeology: GEOPHYSICS, 59:224-232.

\section{Advisory Committee Members and Co-authors}

[1] Dr. Amit Agarwal, Director, APJ Abdul Kalam, Institute of Technology, Dehradun, India

[2] Dr. Michael Patrick Coyle, Chief Executive Officer, Avatar MedVision US LLC, NC, USA

[3] Sathya Govindarajan, Director, Pentagram Research Centre Private Limited, Hyderabad, India

[4] Prashanthi Govindarajan, Director, Pentagram Research Centre Private Limited, Hyderabad, India

[5] Yashaswi Vemuganti, Consultant, Avatar MedVision US LLC, NC, USA

[6] Dr. Jean Claude Perez, IBM European Research Center on Artificial Intelligence, France

[7] Dr. Hindupur Rajasimha, Chief Engineer, Indian Space Research Organization and IDBI (Retd.) 